\title{
Computational Engineering and Science Program at the University of Utah
}

\author{
Carleton DeTar ${ }^{3}$, Aaron L. Fogelson ${ }^{2}$, Chris R. Johnson ${ }^{1}$, \\ Christopher A. Sikorski ${ }^{1}$, and Thanh Truong ${ }^{4}$ \\ 1 School of Computing, University of Utah, Salt Lake City, UT 84112, USA, \\ $\{c r j$, sikorski\}@cs.utah.edu, \\ http://www.cs.utah.edu/ $\{\sim c r j, \sim$ sikorski $\}$ \\ 2 Department of Mathematics, University of Utah, Salt Lake City, UT 84112, USA, \\ fogelson@math.utah.edu, \\ http://www. math. utah.edu/ fogelson \\ 3 Department of Physics, University of Utah, Salt Lake City, UT 84112, USA, \\ detar@physics.utah.edu, \\ http://www.physics.utah.edu/ detar \\ ${ }^{4}$ Department of Chemistry, University of Utah, Salt Lake City, UT 84112, USA, \\ truong@chemistry . utah.edu, \\ http://www. chem.utah.edu/faculty/truong/index.html
}

\begin{abstract}
We summarize the Computational Engineering and Science program at the University of Utah. Program requirements as well as related research areas are outlined. To obtain the MS degree in CES, a student must complete courses and present original research in scientific computing, scientific visualization, mathematical modeling, and the case studies in CES. The outlined research areas include scientific visualization, computational combustion, computational physics, computational chemistry, mathematical and computational biology, and computational medicine.
\end{abstract}

\section{Computational Engineering and Science Program}

The grand computational challenges in engineering and science require for their resolution a new scientific approach. As one report points out, "The use of modern computers in scientific and engineering research and development over the last three decades has led to the inescapable conclusion that a third branch of scientific methodology has been created. It is now widely acknowledged that, along with traditional experimental and theoretical methodologies, advanced work in all areas of science and technology has come to rely critically on the computational approach." This methodology represents a new intellectual paradigm for scientific exploration and visualization of scientific phenomena. It permits a new approach to the solution of problems that were previously inaccessible.

At present, too few researchers have the training and expertise necessary to utilize fully the opportunities presented by this new methodology; more importantly, traditional educational programs do not adequately prepare students to take advantage of these opportunities. 
Too often we have highly trained computer scientists whose knowledge about engineering and sciences is at the college sophomore, or lower, level. Traditional educational programs in each of these areas stop at the sophomore level - or earlier - in the other area. Also, education tends to be ad hoc, on the job and self-taught.

This situation has arisen because the proper utilization of the new methodology requires expertise and skills in several areas that are considered disparate in traditional educational programs. The obvious remedy is to create new programs that do provide integrated training in the relevant areas of science, mathematics, technology, and algorithms. The obvious obstacles are territorial nature of established academic units, entrenched academic curricula, and a lack of resources.

At the University of Utah the School of Computing (located in the College of Engineering), with the Departments of Mathematics and Physics (located in the College of Science) have established a graduate program that we consider a first step towards the modernization of the University's curriculum in what we call "Computational Engineering and Science" (CES). Our goal is to provide a mechanism by which a graduate student can obtain integrated expertise and skills in all areas that are required for the solution of a particular problem via the computational methodology.

We have recently created an M.S. CES degree program. If the M.S. CES program is successfel, we will consider expanding the program to a Ph.D. in CES. Our program is designed mostly for students in the Colleges of Engineering, Mines, and Science. However, in principle any graduate student at the University can participate.

To obtain the MS degree in CES, a student must complete courses and present original thesis research (for the thesis option) in each of the following areas:

\section{Introduction to Scientific Computing \\ II. Advanced Scientific Computation \\ III. Scientific Visualization \\ IV. Mathematical Modeling \\ V. Case Studies in CES \\ VI. Elective course}

\section{Seminar in Computational Engineering and Science}

Of the above items, only V and VII are truly new requirements. Numerical Analysis has been taught in our departments for several years. Mathematical modeling has been spread over a large number of courses in the current Mathematics curriculum; the new course has been designed particularly for the CES program and has replaced one or more other courses in the students' load. The situation in regard to courses I and III in the Computer Science curriculum is very similar.

All courses are designed for first- or second-year science and engineering graduate students who have a knowledge of basic mathematics and computing skills. The most innovative aspect of our CES program is course V., Case Studies in CES. This course consists of presentations by science and engineering faculty from various departments around the campus. These faculty, all active in computationally intensive research, introduce students to their own work over intervals 
of, typically, three weeks. The course provides students with a reasonably deep understanding of both the underlying science and engineering principles involved in the various projects and the practical issues confronting the researchers. It also provide a meeting place for faculty and graduate students engaged in CES activities in various departments throughout campus. In the CES seminar (VII) students are required to report on their own CES activities to their peers.

This approach, then, will serve the students by at once focusing their activities and bringing together in one place several essential components of CES that were previously spread over a larger and less clearly defined part of the existing curriculum. In addition, the program will help students learn to work with researchers in other disciplines and to understand how expertise in another field can help propel their own research forward.

The program is administered by a director and a "steering committee" consisting of two members each from the Departments of Mathematics, Physics and the School of Computing. The committee is advised by a board of faculty active in CES research.

We have already graduated 6 MS students and currently have 21 students participating in the program. We believe that the program will grow to about 30 students by Fall 2004. Our graduates continue their careers in national laboratories, university research laboratories, and technology companies.

\section{Computational Engineering and Science Research}

The University of Utah has a rich pool of faculty who are active in the various areas involved in CES. These include fields such as computational fluid dynamics, physics and chemistry, earthquake simulation, computational medicine, pharmacy, biology, computational combustion, materials science, climate modeling, genetics, scientific visualization and numerical techniques. A few examples of some of the current research in these areas follow.

\subsection{Scientific Visualization}

Common to many of the computational science application areas is the need to visualize model geometry and simulation results. The School of Computing at the University of Utah has been a pioneer and leader in computer graphics and scientific visualization research and education. Some of the first scientific visualizations were invented and displayed here, such as the use of color for finite element analysis and the use of geometrical visualizations of molecules. Utah is home to the Scientific Computing and Imaging (SCI) Institute [1]. SCI Institute researchers have innovated several new techniques to effectively visualize largescale computational fields [2]34/56/7 89].

\subsection{Computational Chemistry}

Prof. Thanh N. Truong from the Department of Chemistry is leading major efforts in collaborations with Dr. Julio Facelli from the University of Utah Center 
for High Performance Computing, Prof. Thom Cheatham from the Medicinal Chemistry Department, Prof. Chuck Wight also from the Chemistry Department, and Prof. James Lewis from Brigham Young University to develop an integrated extendable web-based simulation environment called Computational Science and Engineering On-line (CSEO). A number of graduate students from our CES program are involved in this project. CSEO allows computational scientists to perform research using state-of-the-art tools, querying data from personal or public databases, discuss results with colleagues or attend a workshop, and access resources beyond those available locally from a web browser. CSEO will provide a problem-solving environment for integrating different fields of computational science and engineering, thus facilitating inter-disciplinary research and particularly multi-scale simulations of complex scientific problems. Currently, CSEO supports an integrated environment for multi-scale modeling of complex reacting systems. Specifically, it allows results from fundamental quantum chemistry simulations to be used to calculate thermodynamic and kinetic properties of a chemical reaction, which subsequently are used in the simulation of a combustion reactor. A unique feature of CSEO is in its framework that allows data to flow from one application to another in a transparent manner. Efforts to extend the CSEO environment to computational biology and materials science are also being made. CSEO can be accessed at http://cseo.net.

\subsection{Computational Combustion}

The Center for the Simulation of Accidental Fires and Explosions (CSAFE) (www.csafe.utah.edu), now in its 7 th year, was created at the University of Utah through the Department of Energy's ASCI Program (www.llnl.gov/asci/alliances). C-SAFE focuses on creating a software based simulation system that can accurately simulate the complex interactions between large fires and structures within the fire, especially structures containing explosive materials. This system is designed to effectively use the computational resources available at the National Laboratories which include supercomputers consisting of 1000's of processors [10]. To produce accurate simulations of the chemistry and engineering physics involved, the software requires a combination of fully coupled non-linear solvers, optimization, computational steering, and visualization, along with validation with experimental data 11.

To create such a system, the combined talents of a highly skilled multidisciplinary team are required. Faculty, post-docs, staff members, and students from Chemistry, Chemical Engineering, Mechanical Engineering, and the School of Computing have worked together to produce this software. When it is completed, the software will provide a valuable means of improving and validating the safety of structures and explosive materials when exposed to large fires [12].

\subsection{Mathematical and Computational Biology}

The Mathematics Department at the University of Utah is home to one of the world's largest and most active research groups in Mathematical Biology. The 
work in this group centers on understanding the biological mechanisms that regulate the dynamics of important physiological, biochemical, biophysical, and ecological interactions. A major focus of research, lead by Aaron Fogelson, is in using mathematics and computation to understand how the complex biochemical and biophysical components, especially the fluid dynamics, of platelet aggregation and coagulation interact in hemostasis (normal blood clotting) and thrombosis (pathological blood clotting within blood vessels). This fascinating area has tremendous practical importance because thrombosis is the immediate cause of most heart attacks and strokes. Because the models of clotting are very complex (they involve fluid dynamics, fluid-structure interactions, chemical kinetics, chemical and mass transport), they pose substantial computational challenges, and have required the development of novel numerical methods and software to meet these challenges. This software has been and is being applied to a wide range of biological problems in which fluid flow plays an important role. A second major research area, led by James Keener, involves modeling and three-dimensional computation of electrical waves in cardiac muscle. The goal is to understand normal signal propagation and the coupling between the electrical stimuli and cardiac muscle contraction, to understand the mechanisms underlying the onset of pathological arrythmias, and to understand at the cellular and tissue level how defibrillation works so as to help optimize defibrillation strategies. Some of the other work in the group includes studies of information processing in the primary visual cortex, models of territoriality in interacting animal populations, and studies of invasion of ecosystems by new species.

\subsection{Computational Medicine}

An interdisciplinary team of nationally recognized research centers at Utah involving the Cardiovascular Research and Training Institute (CVRTI), the Scientific Computing and Imaging (SCI) Institute, the Center for Advanced Medical Technology (CAMT), and the Neurosurgery Department are working together to tackle large-scale computational problems in medicine.

Every year, approximately 500,000 people die suddenly because of abnormalities in their hearts' electrical system (cardiac arrhythmias) and/or from coronary artery disease. While external defibrillation units have been in use for some time, their use is limited because it takes such a short time for a heart attack victim to die from insufficient oxygen to the brain. Lately, research has been initiated to find a practical way of implanting electrodes within the body to defibrillate a person automatically upon onset of cardiac fibrillation. Because of the complex geometry and inhomogeneous nature of the human thorax and the lack of sophisticated thorax models, most past design work on defibrillation devices has relied on animal studies. We have constructed a large-scale model of the human thorax, the Utah Torso Model [13 14]15], for simulating both the endogenous fields of the heart and applied current sources (defibrillation devices). Using these computer models, We are also able to simulate the multitude of electrode configurations, electrode sizes, and magnitudes of defibrillation shocks. Given the large number of possible external and internal electrode sites, magnitudes, and configurations, it is a daunting problem to computationally test 
and verify various configurations. For each new configuration tested, geometries, mesh discretization levels, and a number of other parameters must be changed.

Excitation currents in the brain produce an electrical field that can be detected as small voltages on the scalp. By measuring changes in the patterns of the scalp's electrical activity, physicians can detect some forms of neurological disorders. Electroencephalograms, EEGs, measure these voltages; however, they provide physicians with only a snapshot of brain activity. These glimpses help doctors spot disorders but are sometimes insufficient for diagnosing them. For the latter, doctors turn to other techniques; in rare cases, to investigative surgery.

Such is the case with some forms of epilepsy. To determine whether a patient who is not responding to medication has an operable form of the disorder, known as temporal lobe epilepsy, neurosurgeons use an inverse procedure to identify whether the abnormal electrical activity is highly localized (thus operable) or diffused over the entire brain [16 17].

To solve these two bioelectric field problems in medicine, we have created two problem solving environments, SCIRun and BioPSE [18 1920].

Using SCIRun and BioPSE, scientists and engineers are able to design internal defibrillation devices and source models for the epileptic foci, place them directly into the computer model, and automatically change parameters (size, shape and number of electrodes) and source terms (position and magnitude of voltage and current sources) as well as the mesh discretization level needed for an accurate numerical solution. Furthermore, engineers can use the interactive visualization capabilities to visually gauge the effectiveness of their designs and simulations in terms of distribution of electrical current flow and density maps of current distribution.

\subsection{Computational Physics}

Several computational science opportunities are offered by the Physics Department. Students can gain experience in computational physics on platforms ranging from small Beowulf clusters to supercomputers at the national laboratories to the most powerful, special-purpose computers available in the world.

Experimental High Energy Astrophysics. The High Resolution Fly's Eye and the High Energy Gamma Ray research groups collect terabytes of observational data that require processing on high performance parallel computers. Monte Carlo simulations of detector performance and of theories also require intensive computation. The goal of this research is to understand the origins and production mechanisms of the mysterious, energetic particles. Professors Pierre Sokolsky, David Kieda, Paolo Gondolo, Charles Jui, Kai Martens, and Wayne Springer participate in this work.

Computational Astrophysics. Computer simulations allow us to experiment with the Universe at large. For example, by varying the amount of dark matter in a simulated galaxy, and then comparing it to real, observed galaxies, we can 
place constraints on the nature of dark matter. Ben Bromley has run simulations to study the effects of dark matter in colliding galaxies. With Scott Kenyon, Bromley is also working on models for the formation of rocky planets like Earth. Key issues include how rocky planets form, and whether they are common around other stars like our Sun. Recently, Bromley has joined Richard Price in simulating the collision between two black holes. Colliding black holes produce gravity waves which be will detectable by observatories (e.g., LIGO and LISA) in the near future. Only with computer models will we be able to accurately interpret the gravity wave data.

Solving the Strong Interactions. Large scale ab initio simulations of quantum chromodynamics, the theory of interacting quarks and gluons, have proven to be an indispensible guide to our understanding of the masses and structure of the light elementary particles, the decays of heavy mesons, and the cooling of the quark-gluon plasma, which existed in the early universe. Professor Carleton DeTar is carrying out this work.

\subsection{Future Directions}

The field of Computational Engineering and Science holds rich possibilities for future development. The computational paradigm has taken hold in nearly every area in science and engineering. Its use is also becoming more common in many fields outside of science and engineering, such as the social sciences, architecture, business, and history. Its success hinges on researchers' ability and willingness to transcend traditional disciplinary barriers and share expertise and experience with a large group of colleagues who may have been perceived previously as working in unrelated fields. Such boundary-crossing enriches researchers' work by providing new computational opportunities and insights. Building the interdisciplinary spirit will enable the solution of problems that were previously inaccessible. At the University of Utah, we are excited by such prospects and have taken the first step in initiating a mechanism to educate the next generation of scientists and engineers. One concrete future step we are taking is the creation of a Ph.D. degree in Computing that will have a Scientific Computing track. We hope to start admitting students into the new Ph.D. Computing degree program in Fall 2004.

For more information on our CES program visit www.ces.utah.edu or contact Christopher Sikorski (sikorski@cs.utah.edu).

\section{References}

1. Scientific Computing and Imaging Institute; http://www.sci.utah.edu/.

2. Y. Livnat and C.D. Hansen. View dependent isosurface extraction. In IEEE Visualization '98, pages 175-180. IEEE Computer Society, October 1998.

3. Y. Livnat, S.G. Parker, and C.R. Johnson. Fast isosurface extraction methods for large image data sets. In A.N. Bankman, editor, Handbook of Medical Imaging, pages 731-745. Academic Press, San Diego, CA, 2000. 
4. G.L. Kindlmann and D.M. Weinstein. Hue-balls and lit-tensors for direct volume rendering of diffusion tensor fields. In Proceedings of the IEEE Visualization 99, pages 183-189, 1999.

5. G.L. Kindlmann and D.M. Weinstein. Strategies for direct volume rendering of diffusion tensor fields. IEEE Trans. Visualization and Computer Graphics, 6(2):124$138,2000$.

6. C.R. Johnson and A.R. Sanderson. A next step: Visualizing errors and uncertainty. IEEE Computer Graphics and Applications, 23(5):6-10, September/October 2003.

7. J. Kniss, G. Kindlmann, and C. Hansen. Multidimensional transfer functions for interactive volume rendering. IEEE Transactions on Visualization and ComputerGraphics, 8(3):270-285, 2002.

8. D. Breen and R. Whitaker. A level-set approach to $3 \mathrm{~d}$ shape metamorphosis. IEEE Transactions on Visualization and ComputerGraphics, 7(2):173-192, 2001.

9. C.R. Johnson, Y. Livnat, L. Zhukov, D. Hart, and G. Kindlmann. Computational field visualization. In B. Engquist and W. Schmid, editors, Mathematics Unlimited - 2001 and Beyond, volume 2, pages 605-630. Springer-Verlag, 2001.

10. J.D. de St. Germain, J. McCorquodale, S.G. Parker, and C.R. Johnson. Uintah: A massively parallel problem solving environment. In Ninth IEEE International Symposium on High Performance andDistributed Computing, pages 33-41. IEEE, Piscataway, NJ, Nov 2000.

11. R. Rawat, S.G. Parker, P.J. Smith, and C.R. Johnson. Parallelization and integration of fire simulations in the uintah pse. In Proceedings of the Tenth SIAM Conference on Parallel Processing for Scientific Computing, March 12-14 2001.

12. T.C. Henderson, P.A. McMurtry, P.J. Smith, G.A. Voth, C.A. Wight, and D.W. Pershing. Simulating accidental fires and explosions. IEEE Computational Science and Engineering, 2:64-76, 2000.

13. C.R. Johnson, R.S. MacLeod, and P.R. Ershler. A computer model for the study of electrical current flow in the human thorax. Computers in Biology and Medicine, 22(3):305-323, 1992.

14. C.R. Johnson, R.S. MacLeod, and M.A. Matheson. Computational medicine: Bioelectric field problems. IEEE COMPUTER, pages 59-67, October 1993.

15. R.S. MacLeod, C.R. Johnson, and M.A. Matheson. Visualization of cardiac bioelectricity - a case study. In IEEE Visualization '92, pages 411-418, 1992.

16. D.M. Weinstein, L. Zhukov, and C.R. Johnson. Lead-field bases for EEG source imaging. Annals of Biomedical Engineering, 28:1-7, 2000.

17. D. Weinstein, P. Krysl, and C. Johnson. The bioPSE inverse EEG modeling pipeline. In ISGG \%th International Conference on Numerical Grid Generation in Computation Field Simulations, pages 1091-1100, Mississippi State University, 2000. The International Society of Grid Generation.

18. SCIRun: A Scientific Computing Problem Solving Environment. Scientific Computing and Imaging Institute (SCI), http://software.sci.utah.edu/scirun.html, 2002.

19. BioPSE: Problem Solving Environment for modeling, simulation, and visualization of bioelectric fields. Scientific Computing and Imaging Institute (SCI), http://software.sci.utah.edu/biopse.html, 2002.

20. C.R. Johnson, S. Parker, D. Weinstein, and S. Heffernan. Component-based problem solving environments for large-scale scientific computing. Journal on Concurrency and Computation: Practice and Experience, (14):1337-1349, 2002. 